\title{
Update on the diagnosis of GH deficiency in adults
}

Roger Abs

Department of Endocrinology, University Hospital Antwerp, Wilrijkstraat 10, B-2650 Edegem, Belgium

(Correspondence should be addressed to R Abs; Email: rogerabs@ua.ac.be)

\begin{abstract}
GH deficiency (GHD) in adults is associated with considerable morbidity and mortality. The diagnosis of GHD is generally straightforward in children as growth retardation is present; however, in adults, diagnosis of GHD is often challenging. Other markers are therefore needed to identify adults who have GHD and could potentially benefit from GH replacement therapy. Consensus guidelines for the diagnosis and treatment of adult GHD recommend provocative testing of GH secretion for patients who have evidence of hypothalamic-pituitary disease, patients with childhood-onset GHD, and patients who have undergone cranial irradiation or have a history of head trauma. Suspicion of GHD is also heightened in the presence of other pituitary hormone deficits.

Tests for GHD include measurement of the hormone in urine or serum or measurement of stimulated GH levels after administration of various provocative agents. The results of several studies indicate that non-stimulated serum or urine measurements of $\mathrm{GH}$ levels cannot reliably predict deficiency in adults. Although glucagon and arginine tests produce a pronounced GH response with few false positives, the insulin tolerance test (ITT) is currently considered to be the gold standard of the GH stimulation tests available. Unfortunately, the ITT has some disadvantages and questionable reproducibility, which have prompted the development of several new tests for GHD that are based on pharmacological stimuli. Of these, GH-releasing hormone (GHRH) plus arginine and GHRH plus GH-releasing peptide (GHRP) appear to be reliable and practical. Thus, in cases where ITT is contraindicated or inconclusive, the combination of arginine and GHRH is an effective alternative. As experience with this test as well as with GHRH/GHRP-6 accumulates, they may supplant ITT as the diagnostic test of choice.
\end{abstract}

European Journal of Endocrinology 148 S3-S8

\section{Introduction}

The abundance of medical literature describing the detriments of growth hormone deficiency (GHD) in adults underlines the importance of this condition. GHD has widespread unfavourable effects on body composition, lipid metabolism, bone mass, and quality of life. It is associated with significant morbidity and increased mortality (1). GHD in adult patients predominately results from insult to the pituitary or hypothalamus. GH is usually the first hormone to be impacted in the development of hypopituitarism, followed by the gonadotrophins, adrenocorticotrophic hormone, and thyroidstimulating hormone.

Numerous studies have found that treatment with GH replacement therapy provides physical and psychological benefit $(2-5)$. These positive findings prompted investigations aimed at improving the diagnostic criteria for adult GHD. The diagnosis of GHD is straightforward in children because of the cardinal sign of growth retardation. However, diagnosis of adult GHD is challenging, as the pathophysiologic features of adult GHD are non-specific and not exclusive to this hormone deficiency. More precise diagnostic criteria would help determine which patients would benefit most from GH replacement therapy, thereby delaying and perhaps preventing the sequelae associated with this condition.

\section{Indications for biochemical testing of GHD}

Consensus guidelines for the diagnosis and treatment of adult GHD, issued by the Growth Hormone Research Society, recommend testing GH secretion in patients who have evidence of hypothalamic-pituitary disease, patients who have undergone cranial irradiation, and patients with a history of childhood-onset GHD (6). A history of head trauma may also warrant evaluation, as GHD can occur following cerebral injury $(7,8)$. Evidence suggests that the presence of other pituitary hormone deficits is directly associated with GHD. One study revealed a relationship between the degree of hypopituitarism and GH status during an analysis of peak GH responses to an insulin tolerance test (ITT) in 190 non-acromegalic patients with pituitary disease (9). The majority of patients with two or three additional pituitary deficiencies $(90.3 \%)$ had a GH response 
below the accepted normal range compared with only $24.1 \%$ of patients with isolated GHD (Fig. 1).

\section{Diagnostic tests for GHD}

GH status has been assessed by GH measurements using 24-h assays of serum or urinary GH concentrations or more commonly by indirect pharmacological stimuli (e.g. insulin, arginine, levodopa, glucagon) (9, 10). In a study involving 19 patients with hypopituitarism and 30 healthy volunteers, the diagnostic efficacy of

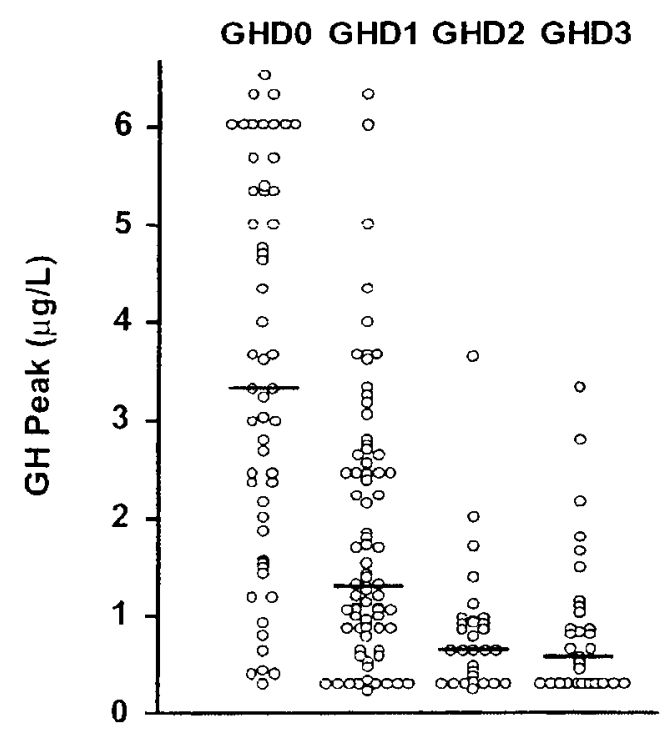

Figure 1 Relationship between the degree of hypopituitarism and GH status. GHD0, isolated GHD; GHD1, GHD plus one pituitary hormone deficit; GHD2, GHD plus two pituitary hormone deficits; GHD3, GHD plus three pituitary hormone deficits. The horizontal bars represent medians. (Reprinted, with permission, from (9).)

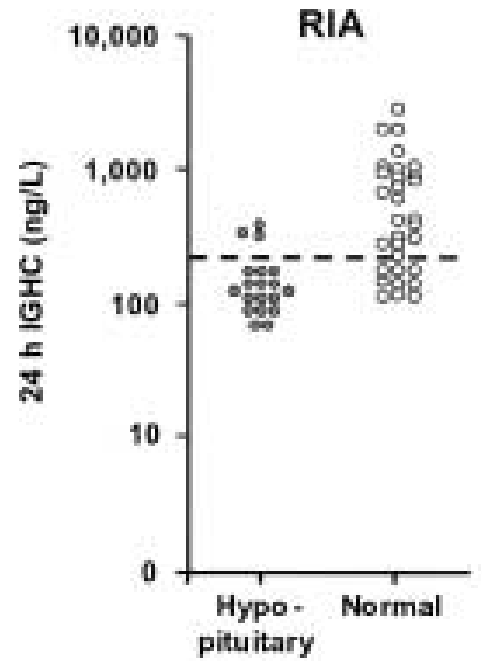

measuring non-stimulated serum GH concentrations in adult GHD was found to be unreliable (11). A comparison of 24-h integrated GH concentrations (IGHC) revealed considerable overlap between patients with GHD and healthy subjects when a radioimmunoassay (RIA) was used, due mainly to the occurrence of undetectable values in both groups. Use of a $\mathrm{GH}$ enzyme-linked immunosorbent assay (ELISA) improved the discriminative power of the test; however, an overlap between the two groups still existed. Although the mean IGHC in healthy subjects was significantly higher than in hypopituitary patients $(852 \pm 131$ vs $97 \pm 28 \mathrm{ng} / \mathrm{l}$, $P=0.0001$ ), the IGHCs from the two groups were not completely distinct/discriminative (Fig. 2). Twenty-six percent of hypopituitary patients had IGHC values within the normal range (i.e. 111-3454 ng/l) while $37 \%$ of healthy subjects had values within the hypopituitary range (i.e. 5-459 ng/l). For both the RIA- and ELISA-derived IGHC, overlap remained evident when patients were stratified by age (11).

Twenty-four hour urinary GH concentrations, measured by RIA, have also been compared in healthy subjects and patients with GHD. In a study by Bates et al. (12), participants were separated into three age groups; 16 to 39 years, 40 to 60 years, and greater than 60 years. Although there was a significant difference in urinary GH levels between patients with GHD and healthy subjects in all three age groups, an overlap was evident, which increased with age (Fig. 3). Thus, urinary GH becomes even more unsuitable with increasing age.

The relationship between insulin-like growth factor-I (IGF-I) and GHD was investigated using a worldwide patient database known as the Pharmacia International Metabolic Study Database (KIMS) (13). In this study, 1034 patients with pre-existing GHD were enrolled, and $88.8 \%$ of patients had two to four pituitary

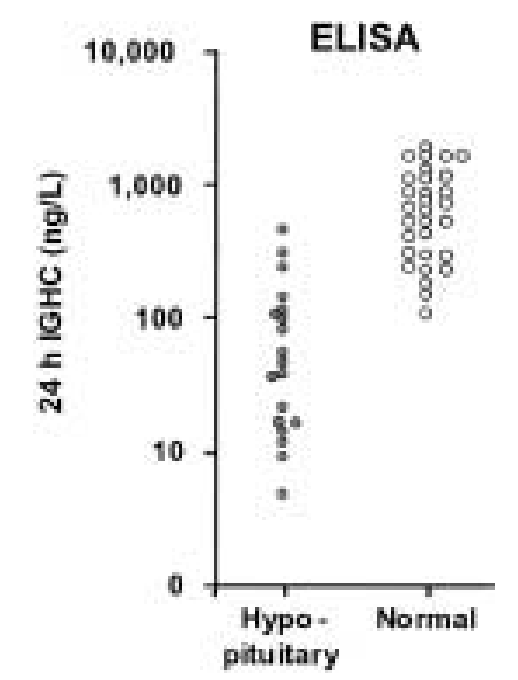

Figure 2 Twenty-four hour IGHC concentrations by RIA and ELISA for hypopituitary and normal subjects. (Reprinted, with permission, from (11).) 
deficiencies in addition to GHD. Approximately $86 \%$ of patients older than 30 years of age had an IGF-I SD score indicative of $\mathrm{GHD}$ versus $42 \%$ of patients older than 50 years of age. The poor discriminative power of IGF-I was even more pronounced in patients deficient in GH only or GH plus one other pituitary hormone. These data suggest that IGF-I is not a reliable marker of GHD in adults, especially in older patients. A low serum IGF-I in conjunction with three or more pituitary hormone deficiencies is, however, highly indicative of GHD with a positive predictive value of $95 \%$ (14).

Since GH and IGF-I concentrations are usually insufficient to diagnose adult GHD, other methods to assess GH secretion are necessary. Provocative tests using pharmacological stimuli for GH secretion include insulin, arginine, glucagon, and GH-releasing hormone (GHRH). Levodopa and clonidine have also been used; however, levodopa is not a potent stimulator of $\mathrm{GH}$ and clonidine has no diagnostic value for GHD in adults (15).

Currently, the ITT is considered the diagnostic test of choice (6). In 23 patients with organic pituitary disease and 35 healthy volunteers, Hoffman et al. (16) evaluated the relative diagnostic merits of four tests, including the peak GH response to ITT, mean 24-h GH concentration, serum IGF-I concentrations, and serum IGF-binding protein-3 (IGFBP-3) concentrations. The ranges of ITTstimulated peak $\mathrm{GH}$ concentrations between the hypopituitary (i.e. $<0.2-3.1 \mu \mathrm{g} / \mathrm{l}$ ) and healthy (i.e. $5.3-42.5 \mu \mathrm{g} / \mathrm{l})$ groups were clearly distinct, whereas mean 24-h GH, IGF-I, and IGFBP-3 concentrations overlapped. Only ITT was able to reliably distinguish patients with GHD from healthy volunteers (Fig. 4) (16).

A GH level of less than $5 \mu \mathrm{g} / \mathrm{l}$ in response to ITT is considered abnormal, and a response of less than $3 \mu \mathrm{g} / \mathrm{l}$ indicates severe GHD. Although the ITT demonstrates good sensitivity, a number of disadvantages exist that hinder its use. ITT is time and labour intensive and has a low degree of reproducibility in healthy adults,

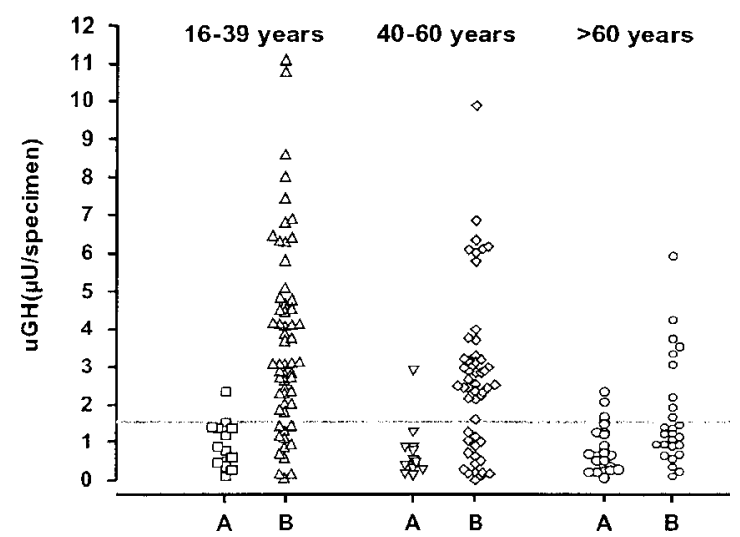

Figure 3 Comparison of urinary $\mathrm{GH}(\mathrm{UGH})$ in patients with known GHD (A) and normal controls (B). The $1.6 \mu \mathrm{U}$ specimen equals sensitivity of $>90 \%$ (horizontal line). $P<0.001$. (Reprinted, with permission, from (12).) which brings its specificity into question. The test has not been analysed in a large group of GHD patients, nor in different age groups. Most control patients are young, non-obese, and do not have many of the co-morbid conditions found in patients with GHD $(17,18)$. In addition, the test may be unpleasant for the patient, and in rare cases, it may result in serious sequelae $(19,20)$. The ITT is contraindicated in patients with a history of ischaemic heart disease and epilepsy; thus, it should be performed in specialised metabolic units.

\section{Diagnostic challenges: factors affecting GH secretory status in adults}

\section{Patient age}

$\mathrm{GH}$ responsiveness and spontaneous $\mathrm{GH}$ secretion, as well as serum IGF-I and IGFBP-3 concentrations, are inversely related to a patient's age as these factors decrease as the patient ages. Toogood et al. (21) compared the effectiveness of spontaneous $\mathrm{GH}$ secretion, the arginine stimulation test, and basal estimates of circulating IGF-I, IGF-II, IGFBP-2, and IGFBP-3 concentrations in detecting GHD in older patients. Patients with non-acromegalic pituitary disease who had a previous peak GH response less than $10 \mu \mathrm{g} / \mathrm{l}$ were compared with healthy subjects. None of the parameters studied demonstrated a clear distinction between patients with GHD and healthy subjects. The median area under the $\mathrm{GH}$ profile (AUCGH) and the peak GH response to arginine were significantly lower in deficient patients than in controls. Although some overlap was evident, a significant relationship was identified between AUCGH and peak GH response to arginine in patients and controls $(r=0.89, \quad P<0.0001 ; \quad r=0.56, \quad P=0.005$ respectively). Serum IGF-I, IGFBP-2, and IGFBP-3 levels were significantly lower in the patients with GHD compared with normative data from an additional 101 patients aged 60 to 87 years (102 vs $142 \mu \mathrm{g} / \mathrm{l}(P<$ $0.0001), 415 \mathrm{vs} 640 \mu \mathrm{g} / \mathrm{l}(P=0.0007)$, and 2.29 vs $2.59 \mathrm{mg} / \mathrm{l}(P=0.009)$, respectively); however, substantial overlap between patients with GHD and healthy volunteers suggested that these measurements were an unsatisfactory means of diagnosis. Thus, while arginine stimulation is more effective than GH markers or spontaneous GH secretion in the diagnosis of GHD in older patients, an overlap between patients with confirmed GHD and healthy subjects still existed with the arginine test. On the other hand, it confirms the different $\mathrm{GH}$ status when comparing patients with GHD with the physiological decline in $\mathrm{GH}$ in normal ageing controls.

\section{Obesity}

Spontaneous GH secretion is generally decreased in obesity, as the capacity of somatotroph cells to secrete $\mathrm{GH}$ appears to be severely impaired (22). Obese patients 

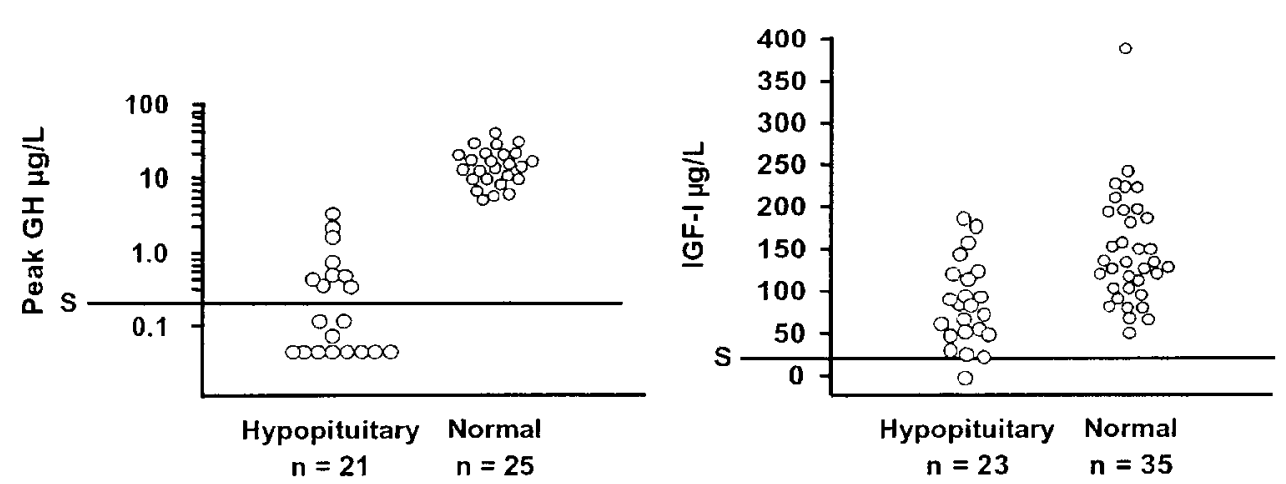

Figure 4 Peak GH response to ITT (left) and IGF-I concentrations (right) in normal and hypopituitary subjects. $\mathrm{S}=$ assay sensitivity for $\mathrm{GH}(0.2 \mathrm{ng} / \mathrm{ml})$ and IGF-I (25 ng/ml). (Adapted, with permission, from (16).)

experience reductions in the half-life of $\mathrm{GH}$ as well as significant decreases in the production and secretion of the hormone. GH responsiveness to pharmacologic stimuli is often blunted in obese patients, as GH secretion is neither elevated following induction of hypoglycaemia nor suppressed by administration of a glucose load (23). In severely obese patients without GHD, peak GH levels after pharmacologic stimulation are often similar to those seen in patients with hypopituitarism (2). In one study, the mean peak response to GHRH was $4.5 \mu \mathrm{g} / \mathrm{l}$ in 14 obese women (mean body weight $105 \mathrm{~kg}$ ) compared with $14.1 \mu \mathrm{g} / \mathrm{l}$ in nine agematched women of normal weight (mean body weight $57 \mathrm{~kg}$ ) (24). Despite alterations in GH production and secretion, IGF-I levels appear to be normal or only slightly diminished in obese patients. Thus, obese patients with reductions in their IGF-I levels should be further evaluated for pituitary dysfunction (22).

\section{New strategies for diagnosing GHD}

The ITT is the gold standard for the diagnosis of GHD, but limitations with this test suggest the need for additional methods to diagnose and monitor GHD. One promising test includes the use of arginine and GHRH, as this combination appears to clearly distinguish patients with GHD from healthy subjects. When administering arginine, a peak GH response of $9 \mu \mathrm{g} / \mathrm{l}$ correlates to a $\mathrm{GH}$ response of $3 \mu \mathrm{g} / \mathrm{l}$ obtained via ITT and indicates severe GHD. Subnormal GH reserve is diagnosed by an ITT response less than $5 \mu \mathrm{g} / \mathrm{l}$, which corresponds to a value of $16.5 \mu \mathrm{g} / \mathrm{l}$ following combined arginine plus GHRH stimulation (17). In a study by Ghigo et al. (25), 73 healthy subjects and 24 patients with hypopituitarism were administered arginine (30 g infused over $30 \mathrm{~min}$ ) and GHRH $(1 \mu \mathrm{g} / \mathrm{kg})(25)$. A differentiation in peak GH responses was noted between healthy subjects (range 16.1$119.0 \mu \mathrm{g} / \mathrm{l}$ ) and patients with hypopituitarism (highest peak value $9.5 \mu \mathrm{g} / \mathrm{l}$ ) (Fig. 5). The sensitivity and specificity of the arginine + GHRH test were $100 \%$ and $95.8 \%$ respectively. Importantly, the test was not influenced by age, as the median GH peak elicited by arginine + GHRH did not significantly differ between older and younger patients (47.6 vs $57.0 \mu \mathrm{g} / \mathrm{l})(25)$.

The ability of arginine + GHRH to distinguish between patients with hypopituitarism and healthy
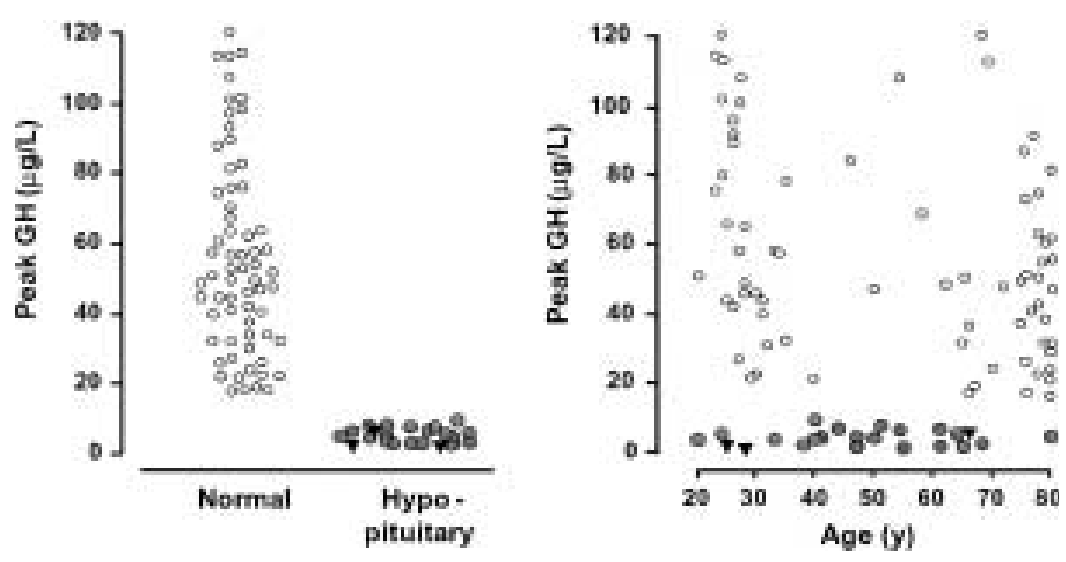

Figure $5 \mathrm{GH}$ response to arginine in patients and controls (০). GH levels in ioiopathic GHD ( $\mathbf{v})$. (Reprinted, with permission, from (25).) 

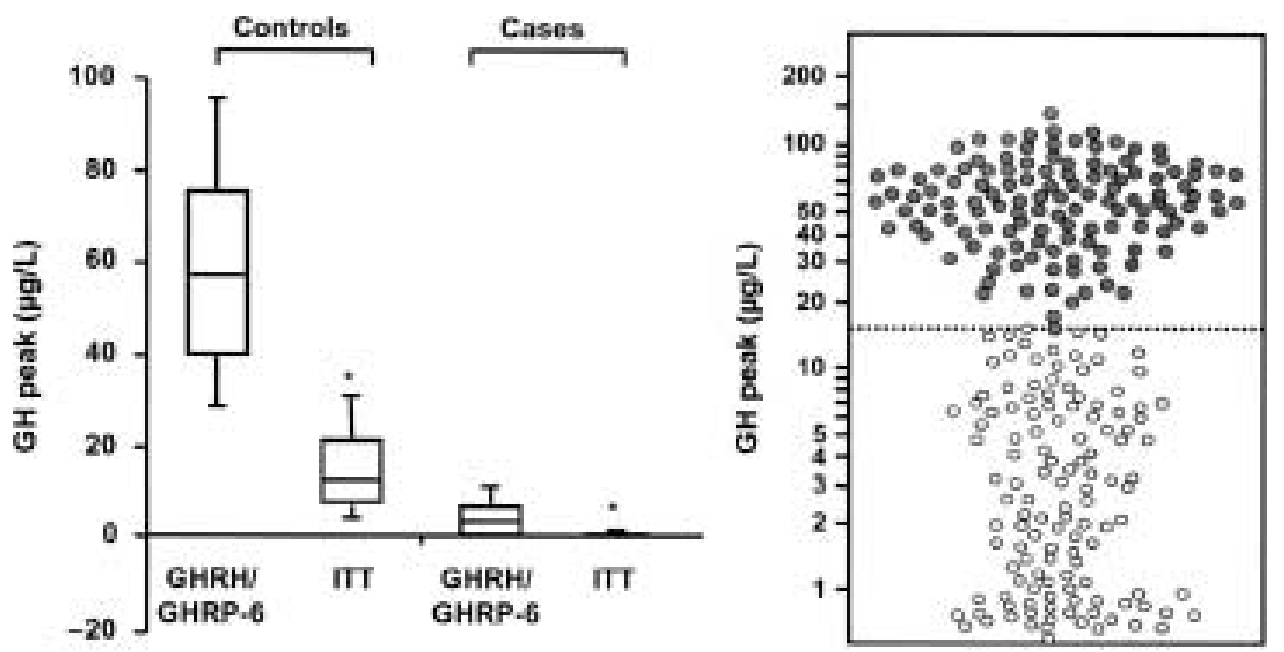

Figure 6 Peak GH secretion after combined administration of GHRH/GHRP-6 versus after ITT in controls and patients with organic pituitary disease (cases). The plot on the right shows the individual GH peaks in controls $(\bullet)$ and patients $(0)$. The distinction between normal and abnormal values appears to occur at $\sim 15 \mu \mathrm{g} / \mathrm{l}$. ${ }^{*} P 0.004$ vs the GHRH/GHRP-6 test. (Adapted, with permission, from (18).)

volunteers was compared with that of ITT (17). The mean peak GH response to ITT in hypopituitary patients was lower than the mean peak response observed with GHRH + arginine (1.5 vs $3.0 \mu \mathrm{g} / \mathrm{l}, P<0.001)$, although there was a positive correlation between $\mathrm{GH}$ response to the two tests $(r=0.61, P<0.001)$. Ten percent of patients with hypopituitarism had GH peaks greater than $5 \mu \mathrm{g} / \mathrm{l}$ after ITT, and an additional $7 \%$ had $\mathrm{GH}$ peaks greater than $3 \mu \mathrm{g} / \mathrm{l}$. Conversely, after GHRH + arginine, all patients had GH peaks less than $16.5 \mu \mathrm{g} / \mathrm{l}$, and $52.5 \%$ had $\mathrm{GH}$ peaks greater than $3 \mu \mathrm{g} / \mathrm{l}(17)$.

Another viable approach to diagnosis is the combination of GHRH with GH-releasing peptide 6 (GHRP-6), an artificial hexapeptide that is considered a very potent and reproducible stimulus of $\mathrm{GH}$ secretion. The specific cut-off value for identifying GHD with the GHRH/GHRP-6 test has not been clearly defined, but values of less than $15 \mu \mathrm{g} / \mathrm{l}$ and less than $17 \mu \mathrm{g} / \mathrm{l}$ have been used $(18,26)$. The diagnostic utility of the GHRH/GHRP-6 combination was recently compared with ITT in 125 patients with hypopituitarism and 125 healthy volunteers (Fig. 6) (18). In this study, GH peaks elicited by GHRH/GHRP-6 were significantly higher than those elicited by ITT in both patients with GHD (4.1 vs $0.5 \mu \mathrm{g} / \mathrm{l}, P<0.0001)$ and healthy volunteers $(59.2$ vs $14.3 \mu \mathrm{g} / \mathrm{l}, P<0.004)$. For the combination test, the differential peak response between healthy subjects and patients with GHD was $55.1 \mu \mathrm{g} / \mathrm{l}$, which was significantly higher than with ITT $(13.8 \mu \mathrm{g} / \mathrm{l}, P<0.001)$, demonstrating the ability of GHRH/GHRP-6 to detect the GHD. Moreover, the results of the GHRH/GHRP-6 test were not influenced by the sex, age, or adiposity in either patients or healthy volunteers (18). Based on these results, the GHRH/GHRP-6 test appears safe and reproducible and free from unpleasant adverse effects, and the combination has no known contraindications to its use. However, cost may be a prohibitive factor.

\section{Conclusions}

The diagnosis of adult GHD should be based on provocative testing of GH secretion, as measurements of serum or urinary GH levels are indeterminate. Although ITT is unpleasant for the patient and complex to administer, requiring close medical supervision, it remains the diagnostic test of choice for adult GHD. In cases in which ITT is contraindicated or inconclusive, a combination of arginine and GHRH is an appropriate alternative. This test is a highly sensitive and specific diagnostic tool and is not influenced by age, making it potentially useful in a broader patient population, taking into account the different cut-off value. GHRH/GHRP-6 also shows considerable promise in this regard. As experience with these tests accumulates, they may supplant ITT as the diagnostic test of choice.

\section{References}

1 Rosen T \& Bengtsson BA. Premature mortality due to cardiovascular disease in hypopituitarism. Lancet $1990336285-288$.

2 De Boer H, Blok GJ \& Van Der Veen EA. Clinical aspects of growth hormone deficiency in adults. Endocrine Reviews 199516 63-86.

3 Monson JP, Abs R, Bengtsson BA, Bennmarker H, FeldtRasmussen U, Hernberg-Stahl E et al. Growth hormone deficiency and replacement in elderly hypopituitary adults. KIMS Study Group and the KIMS International Board. Pharmacia and Upjohn International Metabolic Database. Clinical Endocrinology $200053281-289$

4 Carroll PV, Christ ER, Bengtsson BA, Carlsson L, Christiansen JS, Clemmons D et al. Growth hormone deficiency in adulthood and 
the effects of growth hormone replacement: a review. Growth Hormone Research Society Scientific Committee. Journal of Clinical Endocrinology and Metabolism $1998 \mathbf{8 3} 382-395$.

5 ter Maaten JC, De Boer H, Kamp O, Stuurman L \& Van Der Veen EA. Long-term effects of growth hormone $(\mathrm{GH})$ replacement in men with childhood-onset GH deficiency. Journal of Clinical Endocrinology and Metabolism 199984 2373-2380.

6 Thorner MO, Bengtsson B-Å, Ho KKY, Albertsson-Wikland KM, Christiansen JS, Faglia G et al. Consensus guidelines for the diagnosis and treatment of adults with growth hormone deficiency: summary statement of the Growth Hormone Research Society Workshop on Adult Growth Hormone Deficiency. Journal of Clinical Endocrinology and Metabolism $1998 \mathbf{8 3} 379-381$.

7 Eichler I, Frisch H, Eichler HG \& Soukop W. Isolated growth hormone deficiency after severe head trauma. Journal of Endocrinological Investigation 198811 409-411.

8 Yamanaka C, Momoi T, Fujisawa I, Kikuchi K, Kaji M, Sasaki H et al. Acquired growth hormone deficiency due to pituitary stalk transection after head trauma in childhood. European Journal of Pediatrics 1993152 99-101.

9 Toogood AA, Beardwell CG \& Shalet SM. The severity of growth hormone deficiency in adults with pituitary disease is related to the degree of hypopituitarism. Clinical Endocrinology $1994 \mathbf{4 1}$ $511-516$.

10 Drake WM, Howell SJ, Monson JP \& Shalet SM. Optimizing GH therapy in adults and children. Endocrine Reviews 200122 $425-450$.

11 Reutens AT, Hoffman DM, Leung KC \& Ho KK. Evaluation and application of a highly sensitive assay for serum growth hormone (GH) in the study of adult GH deficiency. Journal of Clinical Endocrinology and Metabolism $1995 \mathbf{8 0} 480-485$.

12 Bates AS, Evans AJ, Jones P \& Clayton RN. Assessment of GH status in adults with GH deficiency using serum growth hormone, serum insulin-like growth factor-I and urinary growth hormone excretion. Clinical Endocrinology 199542 425-430.

13 Abs R, Bengtsson BA, Hernberg-Stahl E, Monson JP, Tauber JP, Wilton P et al. GH replacement in 1034 growth hormone deficient hypopituitary adults: demographic and clinical characteristics, dosing and safety. Clinical Endocrinology 199950 703-713.

14 Molitch ME. Diagnosis of GH deficiency in adults - how good do the criteria need to be? Journal of Clinical Endocrinology and Metabolism 200287 473-476.

15 American Association of Clinical Endocrinologists. AACE clinical practice guidelines for growth hormone use in adults and children. Endocrine Practice 19984 165-173.
16 Hoffman DM, O'Sullivan AJ, Baxter RC \& Ho KK. Diagnosis of growth-hormone deficiency in adults. Lancet $1994 \mathbf{3 4 3}$ 1064-1068.

17 Aimaretti G, Corneli G, Razzore P, Bellone S, Baffoni C, Arvat E et al. Comparison between insulin-induced hypoglycemia and growth hormone (GH)-releasing hormone + arginine as provocative tests for the diagnosis of GH deficiency in adults. Journal of Clinical Endocrinology and Metabolism 199883 1615-1618.

18 Popovic V, Leal A, Micic D, Koppeschaar HP, Torres E, Paramo C et al. GH-releasing hormone and GH-releasing peptide- 6 for diagnostic testing in GH-deficient adults. Lancet $2000 \mathbf{3 5 6}$ 1137-1142.

19 Jones SL, Trainer PJ, Perry L, Wass JA, Bessser GM \& Grossman A. An audit of the insulin tolerance test in adult subjects in an acute investigation unit over one year. Clinical Endocrinology 199441 $123-128$.

20 Shah A, Stanhope R \& Matthew D. Hazards of pharmacological tests of growth hormone secretion in childhood. British Medical Journal 1992304 173-174.

21 Toogood AA, Jones J, O'Neill PA, Thorner MO \& Shalet SM. The diagnosis of severe growth hormone deficiency in elderly patients with hypothalamic-pituitary disease. Clinical Endocrinology 1998 48 569-576.

22 Maccario M, Gauna C, Procopio M, Di Vito L, Rossetto R, Oleandri SE et al. Assessment of GH/IGF-I axis in obesity by evaluation of IGF-I levels and the GH response to GHRH+arginine test. Journal of Endocrinological Investigation 199922 424-429.

23 Scacchi M, Pincelli AI \& Cavagnini F. Growth hormone in obesity. International Journal of Obesity and Related Metabolic Disorders 199923 260-271.

24 Kopelman PG, Noonan K, Goulton R \& Forrest AJ. Impaired growth hormone response to growth hormone releasing factor and insulin-hypoglycaemia in obesity. Clinical Endocrinology $19852387-94$.

25 Ghigo E, Aimaretti G, Gianotti L, Bellone J, Arvat E \& Camanni F. New approach to the diagnosis of growth hormone deficiency in adults. European Journal of Endocrinology $1996134352-356$.

26 Mahajan T \& Lightman SL. A simple test for growth hormone deficiency in adults. Journal of Clinical Endocrinology and Metabolism 200085 1473-1476.

Received 6 December 2002

Accepted 20 December 2002 